PROCEEDINGS OF THE

AMERICAN MATHEM ATICAL SOCIETY

Volume 49, Number 2, June 1975

\title{
AN EXTENSION OF THE DENJOY INTEGRAL
}

\section{JAMES FORAN}

ABSTR ACT. The purpose of this paper is to exhibit an additive class of continuous functions such that each function of the class satisfies Lusin's condition (N). Restricting this class to those functions which are approximately derivable almost everywhere produces a class of primitives which is more extensive than the class of ACG functions, the primitives for the wide sense Denjoy integral.

Let $\mathcal{F}$ be any additive class of continuous functions such that each function $F \in \mathcal{F}$ satisfies Lusin's condition (N). Let $\mathcal{D}$ be an additive class of continuous functions derivable in a sense which is compatible with the ordinary derivative $F^{\prime}(x)$; i.e., $D F(x)=F^{\prime}(x)$ at almost every point where $F^{\prime}(x)$ exists. For each pair of functions $F, G \in \mathscr{T}$, suppose that $D$ satisfies $D(F-G)=D F-D G$ almost everywhere. Then $\mathcal{F} \cap \mathcal{D}$ can be taken as a class of primitives and the $\mathfrak{F} \mathfrak{D}$-integral can be defined by

$$
\mathfrak{F D} \int_{a}^{b} f(x) d x=F(b)-F(a)
$$

where $D F(x)=f(x)$ almost everywhere on $[a, b]$. The uniqueness of the integration is implied by the following theorem:

If a continuous function $F$ satisfies Lusin's condition $(\mathrm{N})$ on an interval $I$ and $F^{\prime}(x)$ is nonnegative at almost every point $x$ where $F^{\prime}(x)$ exists, then $F$ is monotone nondecreasing on $I$ (cf. [1, pp. 285-286]).

Then if $D F=D G$ almost everywhere on $I$, it follows that $D(F-G)=$ $D F-D G=0=D(G-F)$ almost everywhere on $I$ and thus $F-G$ is a constant (the theorem implies that both $F-G$ and $G-F$ are monotone nondecreasing). Consequently

$$
\mathcal{F D} \int_{a}^{b} f(x) d x=F(b)-F(a)=G(b)-G(a)
$$

and the integration yields a unique value.

In what follows $D F(x)$ will be the approximate derivative: $F_{\text {ap }}^{\prime}\left(x_{0}\right)=L$

Received by the editors February $28,1974$.

AMS (MOS) subject classifications (1970). Primary 26A39; Secondary 26A45.

Copyright $\odot 1975$, American Mathematical Society 
if there is a set $A_{x_{0}}$ so that the density of $A_{x_{0}}$ at $x_{0}$ equals 1 and

$$
\lim _{x \rightarrow x_{0}} \frac{F(x)-F\left(x_{0}\right)}{x-x_{0}}=L
$$

when the limit is taken with respect to points $x$ in $A_{x_{0}}$. T will consist of the continuous functions which are approximately derivable almost everywhere. (Alternative differentiation processes could be considered here and hopefully one can be found that includes every function in $\mathcal{F}_{\text {.) }}$

The following definitions are preliminary to defining the class $\mathcal{F}$.

Definition 1. Given a natural number $N$ and a set $E$, a function $f$ will be said to be $B(N)$ on $E$ if there is a number $M<\infty$ such that for any sequence $I_{1}, \ldots, I_{k}, \ldots$ of nonoverlapping intervals with $I_{k} \cap E \neq \varnothing$, there exist intervals $J_{k n^{\prime}} n=1,2, \cdots, N$, such that

$$
B\left(f ; E \cap \bigcup_{k} I_{k}\right) \subset \bigcup_{k} \bigcup_{n=1}^{N} I_{k} \times J_{k n} \text { and } \sum_{k} \sum_{n=1}^{N}\left|J_{k n}\right|<M \text {. }
$$

(Here, $B(f ; X)$ is the graph of $F$ on the set $X$.)

Definition 2, Given a natural number $N$ and a set $E$, a function $f$ will be said to be $A(N)$ on $E$ if for every $\epsilon>0$ there is a $\delta>0$ such that if $I_{1}, \ldots, I_{k}, \ldots$ are nonoverlapping intervals with $E \cap I_{k} \neq \varnothing$ and $\Sigma_{k}\left|I_{k}\right|<$ $\delta$, then there exist intervals $J_{k n}, n=1,2, \ldots, N$, such that

$$
B\left(f ; E \cap \bigcup_{k} I_{k}\right) \subset \bigcup_{k} \bigcup_{n=1}^{N} I_{k} \times J_{k n} \text { and } \sum_{k} \sum_{n=1}^{N}\left|J_{k n}\right|<\epsilon \text {. }
$$

The class $\mathcal{F}$ will consist of all continuous functions $F$ defined on closed interval $I$ for which there exist a sequence of sets $E_{n}$ and natural numbers $N_{n}$ such that $I=\bigcup_{n} E_{n}$ and $F$ is $A\left(N_{n}\right)$ on $E_{n}$.

From Definitions 1 and 2 it follows that:

(i) In the event that $N=1$ or $E$ is an interval, if $F$ is a continuous function satisfying $A(N)[B(N)]$ on $E$, then $f$ is absolutely continuous [of bounded variation] on $E$.

(ii) If a continuous function $f$ satisfies $A(N)[B(N)]$ on $E$, then $f$ satisfies $A(N)[B(N)]$ on $\bar{E}=$ the closure of $E$, and on any subset of $\bar{E}$.

(iii) If a function $f$ satisfies $A(N)$ on $E$ and $Z$ is a set of Lebesgue measure 0 contained in $E$, then $\Lambda(B(f ; Z)$ ) $=0$ (where $\Lambda(x)$ is the linear measure of $X$ ). Consequently, the Lebesgue measure of $f(Z)$ is also 0 .

(iv) If a function $f$ is $B(N)$ on $E$ with $|E|<\infty$ then $\Lambda(B(f ; E))<\infty$. 
(v) If a function $f$ is continuous and satisfies $A(N)$ on a bounded set $E$ then $f$ satisfies $B(N)$ on $E$.

(vi) If a function $f_{1}$ satisfies $A(N)[B(N)]$ on $E$ and a function $f_{2}$ satisfies $A\left(N^{\prime}\right)\left[B\left(N^{\prime}\right)\right]$ on $E$ then every linear combination of $f_{1}$ and $f_{2}$ satisfies $A\left(N \cdot N^{\prime}\right)\left[B\left(N \cdot N^{\prime}\right)\right]$ on $E$. If $f_{1}$ and $f_{2}$ are bounded on $E$ then $f_{1} \cdot f_{2}$ also satisfies $A\left(N \cdot N^{\prime}\right)\left[B\left(N \cdot N^{\prime}\right)\right]$ on $E$.

Proof of (i). If $N=1$ then Definition 1 is equivalent to the definition of absolute continuity on a set $E$ and Definition 2 is equivalent to the definition of bounded variation on a set $E$. If $E$ is an interval, since $f$ is continuous, the graph of $f$ is connected and $\bigcup_{n=1}^{N} J_{k n}$ obtained by the definition of $A(N)[B(N)]$ is an interval (for each $k$ ). Consequently

$$
\sum_{k} O\left(f ; I_{k}\right) \leq \sum_{k}\left(\sum_{n=1}^{N}\left|J_{k n}\right|\right)<\epsilon
$$

whenever $\Sigma\left|I_{k}\right|<\delta$, where $O(f ; I)$ is the oscillation of $f$ on $I$. Thus $f$ is absolutely continuous on the interval $E\left[\Sigma_{k} O\left(f ; I_{k}\right) \leq \Sigma_{k}\left(\Sigma_{n=1}^{N}\left|J_{k n}\right|\right)<M<\right.$ $\infty$ and $f$ is of bounded variation on the interval $E]$.

Proof of (ii). If $f$ is continuous and satisfies $A(N)[B(N)]$ on $E$, it is sufficient that the definitions be satisfied for every finite sequence of closed intervals $I_{k}$. But then $B\left(f ; E \cap \bigcup_{I_{k}}\right) \subset \bigcup_{k} \bigcup_{n=1}^{N} I_{k} \times J_{k n}$ holds true if $E$ is replaced by $\bar{E}$. Thus $f$ satisfies $A(N)[B(N)]$ on $\bar{E}$ and also on any subset of $\bar{E}$.

Proof of (iii). Let $Z \subset E$ be a set of measure 0 and $f$ be $A(N)$ on $E$. For any $\epsilon>0$ there is a $\delta>0$ such that if $I_{1}, \ldots, I_{k}, \ldots$ is a sequence of nonoverlapping intervals with $I_{k} \cap E \neq \varnothing$ and $\Sigma\left|I_{k}\right|<\delta$ there exist $J_{k n^{\prime}}$ $n=1,2, \cdots, N$, such that

$$
B\left(f ; E \cap \bigcup I_{n}\right) \subset \bigcup_{k} \bigcup_{n=1}^{N} I_{k} \times J_{k n} \text { and } \sum_{k} \sum_{n=1}^{N}\left|J_{k n}\right|<\epsilon .
$$

Select a sequence of intervals $I_{1}, \ldots, I_{k}, \cdots$ such that $Z \subset \cup I_{k}$ and $\Sigma\left|I_{k}\right|<\min (\epsilon, \delta)$. It follows that

$$
\begin{aligned}
\sum_{k, n} \operatorname{diam}\left(I_{k} \times J_{k n}\right) & \leq \sum_{k, n}\left|I_{k}\right|+\left|J_{k n}\right|=N \cdot \sum_{k}\left|I_{k}\right|+\sum_{k, n}\left|J_{k n}\right| \\
& <N \delta+\epsilon \leq(N+1)_{\epsilon} .
\end{aligned}
$$

Since $\epsilon$ is arbitrary, it follows that $\Lambda(B(f ; Z))=0$.

Proof of (iv). Given $\epsilon>0$, let $I_{1}, \ldots, I_{k}, \ldots$ be a sequence of non- 
overlapping intervals containing $E$ such that $\left|I_{k}\right|<\epsilon$ and $\Sigma\left|I_{k}\right|-|E|<\epsilon$. Then there are intervals $J_{k n}, n=1,2, \cdots, N$, such that

$$
B(f ; E) \subset \bigcup_{k} \bigcup_{n=1}^{N} I_{k} \times J_{k n} \text { and } \sum_{k} \sum_{n=1}^{N}\left|J_{k n}\right|<M<\infty,
$$

where $M$ is the bound dependent on $f$. Each interval $J_{k n}$ can be written as the union of at most $\left[\left|J_{k n}\right| /\left|I_{k}\right|\right]+1$ intervals $J_{k n m}$ of length $\left|I_{k}\right|$ (where $[x]$ is the greatest integer less than $x$ ). But then

$$
\begin{aligned}
\sum \operatorname{diam}\left(I_{k} \times J_{k n m}\right) & \leq \sum_{n, k, m}\left(\left|I_{k}\right|+\left|J_{k n m}\right|\right) \leq \sum_{k, n} 2\left(\frac{\left|J_{k n}\right|}{\left|I_{k}\right|}+1\right)\left|I_{k}\right| \\
& \leq \sum_{k, n} 2\left(\left|J_{k n}\right|+\left|I_{k}\right|\right) \leq 2(M+N(|E|+\epsilon)) .
\end{aligned}
$$

Since $\epsilon$ is arbitrary, $\Lambda(B(f ; E)) \leq 2 M+2 N|E|<\infty$.

Proof of (v). From (ii) and the hypotheses of (v), $f$ satisfies $A(N)$ on the compact set $\bar{E}$. For $\epsilon=1$ let $\delta>0$ be the $\delta$ guaranteed by the definition of $A(N)$. For each $x \in E$ let $U(x)$ be the open interval $(x-\delta / 2, x+\delta / 2)$. Since the $U(x)$ form an open cover of $\bar{E}$ there is a finite subcover $U_{1}(x), \ldots, U_{M}(x)$. If $|f(x)|<K$ on $\bar{E}$ then, whenever $I_{1}, \ldots, I_{k}, \ldots$ are pairwise nonoverlapping intervals with $I_{k} \cap E \neq \varnothing$, let $\left\{I_{m}\right\}$ be those intervals contained in $U_{m}(x)$. There are $J_{m n}, n=1,2, \cdots, N$, such that

$$
B\left(f ; E \cap \bigcup_{m} I_{m}\right) \subset \bigcup_{m} \bigcup_{n=1}^{N} I_{m} \times J_{m n} \text { and } \sum_{m} \sum_{n=1}^{N}\left|J_{m n}\right|<1 \text {. }
$$

For the intervals $I_{k}$ which are contained in no $U_{m}(x)$ let $J_{k}^{n}=[-K, K]$ and note that there are at most $2 M$ such intervals $I_{k}$. Then

$$
B\left(f ; E \cap \bigcup_{k} I_{k}\right) \subset \bigcup_{k} \bigcup_{n=1}^{N} J_{k n} \times I_{k} \text { and } \sum_{k} \sum_{n=1}^{N}\left|J_{k n}\right| \leq 2 K \cdot 2 M+M<\infty \text {. }
$$

Proof of (vi). If $f_{1}$ and $f_{2}$ are two functions and

$$
B\left(f_{1} ; E \cap \cup I_{k}\right) \subset \bigcup_{k} \bigcup_{n=1}^{N} I_{k} \times J_{k n}
$$

and

$$
B\left(f_{2} ; E \cap \bigcup_{k}\right) \subset \bigcup_{k} \bigcup_{n^{\prime}=1}^{N^{\prime}} I_{k} \times J_{k n^{\prime}}
$$


then if

$$
J_{k n n^{\prime}}=\left[a_{k n}+a_{k n^{\prime}}, b_{k n}+b_{k n^{\prime}}\right] \text {, }
$$

where $J_{k n}=\left[a_{k n^{\prime}}, b_{k n}\right]$ and $J_{k n^{\prime}}=\left[a_{k n}, b_{k n^{\prime}}\right]$, it follows that

$$
B\left(f_{1}+f_{2} ; E \cap \bigcup I_{k}\right) \subset \bigcup_{k} \bigcup_{n=1}^{N} \bigcup_{n^{\prime}=1}^{N^{\prime}} I_{k} \times J_{k n n^{\prime}} .
$$

Since $\left|J_{k n n^{\prime}}\right|=\left|J_{k n}\right|+\left|J_{k n^{\prime}}\right|$ it follows that

$$
\sum_{k, n, n^{\prime}}\left|J_{k n n^{\prime}}\right|=N^{\prime} \sum_{k, n}\left|J_{k n}\right|+N \sum_{k, n}\left|J_{k n^{\prime}}\right| \text {. }
$$

Thus if $f_{1}$ and $f_{2}$ are $B(N)$ and $B\left(N^{\prime}\right)$ respectively on $E$ then $f_{1}+f_{2}$ is $B\left(N \cdot N^{\prime}\right)$ on $E$. In the case where $f_{1}$ is $A(N)$ and $f_{2}$ is $A\left(N^{\prime}\right)$ on $E$, given $\epsilon>0$, let $\epsilon_{1}=\epsilon / 2 N^{\prime}$ and $\epsilon_{2}=\epsilon / 2 N$. Let $\delta_{1}$ be the $\delta$ determined by $\epsilon_{1}$ and the fact that $f_{1}$ satisfies $A(N)$ on $E$; let $\delta_{2}$ be the $\delta$ determined by $\epsilon_{2}$ and the fact that $f_{2}$ satisfies $A\left(N^{\prime}\right)$ on $E$; let $\delta_{0}=\min \left(\delta_{1}, \delta_{2}\right)$. Then, if $I_{1}, \ldots, I_{k}, \ldots$ are pairwise nonoverlapping intervals with $I_{k} \cap E \neq \varnothing$ and $\Sigma_{k}\left|I_{k}\right|<\delta_{0}$, there are $J_{k n^{\prime}}, n=1,2, \ldots, N$, and $J_{k n^{\prime}}, n^{\prime}=1,2, \ldots, N^{\prime}$, such that

$$
\begin{aligned}
& B\left(f_{1} ; E \cap \bigcup_{k} I_{k}\right) \subset \bigcup_{k} \bigcup_{n=1}^{N} I_{k} \times J_{k n} \text { and } \sum_{k, n}\left|J_{k n}\right|<\epsilon_{1}, \\
& B\left(f_{2} ; E \cap \bigcup_{k} I_{k}\right) \subset \bigcup_{k} \bigcup_{n^{\prime}=1}^{N^{\prime}} I_{k} \times J_{k n^{\prime}} \text { and } \sum_{k, n^{\prime}}\left|J_{k n^{\prime}}\right|<\epsilon_{2} .
\end{aligned}
$$

Consequently

$$
B\left(f_{1}+f_{2} ; E \cap \bigcup_{k} I_{k}\right) \subset \bigcup_{k} \bigcup_{n=1}^{N} \bigcup_{n^{\prime}=1}^{N^{\prime}} J_{k n n^{\prime}}
$$

and

$$
\sum_{k, n, n^{\prime}}\left|J_{k n n^{\prime}}\right|<N^{\prime} \epsilon_{1}+N \epsilon_{2}<\epsilon .
$$

Thus $f_{1}+f_{2}$ is $A\left(N \cdot N^{\prime}\right)$ on $E$. Since it is apparent that $k_{1} f_{1}$ belongs to $A(N)$ and $k_{2} f_{2}$ belongs to $A\left(N^{\prime}\right)$ (respectively $B(N)$ and $B\left(N^{\prime}\right)$ ) it follows that linear combinations of $f_{1}$ and $f_{2}$ belong to $A\left(N \cdot N^{\prime}\right)\left[B\left(N \cdot N^{\prime}\right)\right]$. In the event that $f_{1}$ and $f_{2}$ are bounded, it is sufficient to consider that $0 \leq$ $f_{i}(x) \leq A<\infty$. Then if 


$$
J_{k n n^{\prime}}=\left[a_{k n} \cdot a_{k n^{\prime}}, b_{k n} \cdot b_{k n^{\prime}}\right]
$$

when $J_{k n}=\left[a_{k n^{\prime}}, b_{k n}\right]$ and $J_{k n^{\prime}}=\left[a_{k n}, b_{k n}{ }^{\prime}\right]$ it follows that

$$
\begin{aligned}
\left|J_{k n n^{\prime}}\right| & =b_{k n} \cdot b_{k n^{\prime}}-a_{k n} \cdot a_{k n^{\prime}} \\
& =b_{k n}\left(b_{k n^{\prime}}-a_{k n^{\prime}}\right)+a_{k n^{\prime}}\left(b_{k n}-a_{k n^{\prime}}\right) \leq A\left|J_{k n}\right|+A\left|J_{k n^{\prime}}\right| .
\end{aligned}
$$

The proof that $f_{1} \cdot f_{2}$ is in $A\left(N \cdot N^{\prime}\right)\left[B\left(N \cdot N^{\prime}\right)\right]$ on $E$ proceeds similarly to the proof for the sum of $f_{1}$ and $f_{2}$.

One may note that each function $F$ belonging to $\mathcal{F}$ on an interval $I$ is absolutely continuous on some subinterval of $I$. (This follows from the definition of $\mathcal{F}$, (i), (ii), and the Baire category theorem.)

If $F$ and $G$ belong to $\mathcal{F}$ on $I$, there exist sets $\left\{E_{n}\right\}_{n=1}^{\infty},\left\{E_{k}^{\prime}\right\}_{k=1}^{\infty}$ and natural numbers $\left\{N_{n}\right\}_{n=1}^{\infty},\left\{N_{k}^{\prime}\right\}_{k=1}^{\infty}$ so that $F$ is $A\left(N_{n}\right)$ on $E_{n}, G$ is $A\left(N_{k}^{\prime}\right)$ on $E_{k}^{\prime}$ and $I=\bigcup_{n} E_{n}=\bigcup_{k} E_{k}^{\prime}$. From (vi) it follows that $F+G$ is $A\left(N_{n} \cdot N_{k}^{\prime}\right)$ on $E_{n} \cap E_{k}^{\prime}$. Since $I=\bigcup_{n, k}\left(E_{n} \cap E_{k}^{\prime}\right)$ it follows that $F+G$ belong to $\mathcal{F}$ on $I$. Since (iii) implies that each function $F$ belonging to $\mathcal{F}$ on $I$ also satisfies Lusin's condition $(N)$ on $I$, it follows that $\mathcal{F}$ is an additive class of functions each of which satisfies Lusin's condition (N). From the preliminary remarks one concludes that $\mathcal{F} \cap \mathcal{I}$ can be taken as a class of primitives on an interval $I$.

In conclusion a member of $\mathcal{F}$ which is not ACG is constructed.

Example. 'Let $I=[0,1]$ and let $C$ denote the Cantor ternary set; i.e., $C=\left\{x \mid x=\Sigma c_{i} / 3^{i}\right.$ with $c_{i}=0$ or $c_{i}=2$ for each $\left.i\right\}$. Each point $x \in C$ is uniquely represented by $\Sigma_{c_{i}}(x) / 3^{i}$. Let $\left\{j_{k}\right\}$ be a strictly increasing sequence of natural numbers and set $j_{0}=0$. Let $b(i)=j_{k-1}$ when $j_{k-1} \leq i$ $<j_{k}$, and define $F$ on $C$ by $F\left(\Sigma_{c_{i}} / 3^{i}\right)=\Sigma_{c_{h(i)}} / 3^{i}$. Then $F$ is continuous on $C$ and, by extending $F$ linearly on each interval contiguous to $C$, one has $F$ defined and continuous on $[0,1]$. Consider an interval $I$ such that In $C \neq \varnothing$ and $3^{-m-1} \leq|I|<3^{-m}$ for a natural number $m$. Since $|I|<3^{-m}$, there exist $c_{1}, c_{2}, \ldots, c_{m}$ such that if $x \in \operatorname{I\cap } C$ then $c_{i}(x)=c_{i}, i=1,2$, $\cdots, m$. Let $A=\sum_{i=1}^{m} c_{i} / 3^{i}$ and suppose that $b(m)=j_{k-1}$. Let $J_{1}$ and $J_{2}$ be two intervals defined by

$$
\begin{aligned}
& J_{1}=\left[F(A), F\left(A+\sum_{i>j_{k}} 2 / 3^{i}\right)\right], \\
& J_{2}=\left[F\left(A+2 / 3^{j}\right), F\left(A+\sum_{i \geq j_{k}} 2 / 3^{i}\right)\right] .
\end{aligned}
$$


Then $F(I \cap C) \subset J_{1} \cup J_{2}$ and $\left|J_{1}\right|=\left|J_{2}\right|=3^{-j} k \leq 3^{-m}<3|I|$. It now follows that $F$ is $A(2)$ on $C$. For if $\left\{I_{n}\right\}$ is a sequence of nonoverlapping intervals with $I_{n} \cap C \neq \varnothing$ and $\Sigma\left|I_{n}\right|<\epsilon / 6$, then there exist intervals $J_{1 n^{\prime}} J_{2 n}$ such that

$$
B\left(F ; C \cap \bigcup_{n} I_{n}\right) \subset \bigcup_{n} \bigcup_{k=1}^{2} I_{n} \times J_{k n}
$$

and

$$
\sum_{n} \sum_{k=1}^{2}\left|J_{k n}\right| \leq \sum_{n} \sum_{k=1}^{2} 3\left|I_{n}\right| \leq 6 \sum_{n}\left|I_{n}\right|<\epsilon .
$$

Since $F$ is $A(1)$ (absolutely continuous) on each interval contiguous to $C$, it follows that $F \in \mathcal{F}$. Appropriate choice of the numbers $j_{k}$ results in an $F$ which is not ACG. In particular, if $j_{k+1}$ is chosen so large that $n_{k}=$ $j_{k+1}-j_{k}-1$ satisfies $2^{n} k-1>k \cdot 3^{j} k$, then $F$ is not ACG. In order to show this, it suffices to show that $F$ is of unbounded variation on every portion of $C$ (cf. [1, p. 233]). To see this, let $c_{i}$ be 0 or 2 for $i=1,2$, $\cdots, j_{k}$. Let $a=\sum_{i=1}^{j} c_{i}$ and $b=a+\sum_{i=j_{k}+1}^{\infty} 2 / 3^{i}$. Then there is a sequence of $2^{n k}$ numbers $x$ in $I=[a, b]$ at which $F(x)=F(a)$ or $F(x)=F(b)$ in alternating fashion; namely, all the numbers $x$ satisfying $c_{i}(x)=c_{i}, i=1,2, \cdots, j_{k}$, and either $c_{i}(x)=0$ for each $i \geq j_{k+1}$ or $c_{i}(x)=2$ for each $i \geq j_{k+1}$. Since $F(b)-F(a)$ $=3^{-j_{k}}$ it follows that the variation of $F$ on $I \cap C$ is greater than $\left(2^{n_{k}}-1\right) 3^{-j_{k}}>k$. Because any portion of $C$ contains such an interval $I$, it follows that $F$ is of unbounded variation on each portion of $C$ and hence is not ACG.

\section{REFERENCE}

1. S. Saks, Theory of the integral, 2nd rev. ed., Monografie Mat., vol. VII, PWN, Warsaw, 1937.

DEP ARTMENT OF MATHEMATICS, UNIVERSITY OF WISCONSIN-MIL WAUKEE, MILWAUKEE, WISCONSIN 53201

Current address: Department of Mathematics, University of Missouri-Kansas City, Kansas City, Missouri 64110 